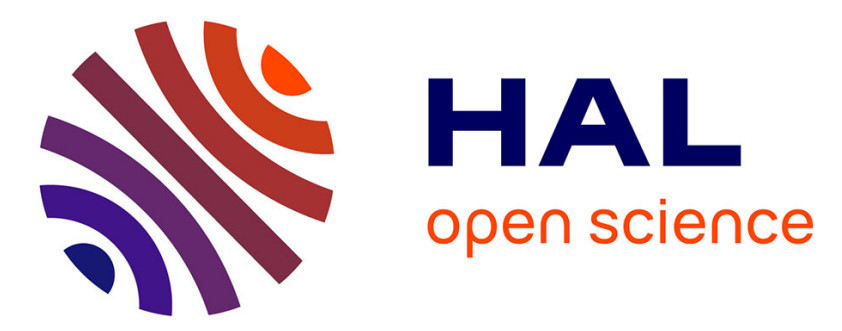

\title{
A General Framework for Curve and Surface Comparison and Registration with Oriented Varifolds
}

\author{
Irène Kaltenmark, Benjamin Charlier, Nicolas Charon
}

\section{To cite this version:}

Irène Kaltenmark, Benjamin Charlier, Nicolas Charon. A General Framework for Curve and Surface Comparison and Registration with Oriented Varifolds. 2017 IEEE Conference on Computer Vision and Pattern Recognition (CVPR), Jul 2017, Honolulu, United States. 10.1109/CVPR.2017.487 . hal-01817514

\section{HAL Id: hal-01817514 \\ https://hal.science/hal-01817514}

Submitted on 18 Jun 2018

HAL is a multi-disciplinary open access archive for the deposit and dissemination of scientific research documents, whether they are published or not. The documents may come from teaching and research institutions in France or abroad, or from public or private research centers.
L'archive ouverte pluridisciplinaire HAL, est destinée au dépôt et à la diffusion de documents scientifiques de niveau recherche, publiés ou non, émanant des établissements d'enseignement et de recherche français ou étrangers, des laboratoires publics ou privés. 


\title{
A general framework for curve and surface comparison and registration with oriented varifolds
}

\author{
Irène Kaltenmark \\ CMLA, ENS Cachan, CNRS, \\ Université Paris-Saclay, 94235, Cachan, France \\ irene.kaltenmarkecmla.ens-cachan.fr
}

\author{
Benjamin Charlier \\ IMAG, CNRS, \\ Université de Montpellier \\ benjamin. charliereumontpellier.fr
}

\author{
Nicolas Charon \\ Center of Imaging Sciences, Johns Hopkins University \\ charonecis.jhu.edu
}

\begin{abstract}
This paper introduces a general setting for the construction of data fidelity metrics between oriented or nonoriented geometric shapes like curves, curve sets or surfaces. These metrics are based on the representation of shapes as distributions of their local tangent or normal vectors and the definition of reproducing kernels on these spaces. The construction, that combines in one common setting and extends the previous frameworks of currents and varifolds, provides a very large class of kernel metrics which can be easily computed without requiring any kind of parametrization of shapes and which are smooth enough to give robustness to certain imperfections that could result e.g. from bad segmentation. We then give a sense, with synthetic examples, of the versatility and potentialities of such metrics when used in various problems like shape comparison, clustering and diffeomorphic registration.
\end{abstract}

\section{Introduction}

Context. Shape analysis has become one of the central problem in many recent applications that ranges from automatic object recognition in computer vision and robotics to the field of medical imaging where more and more advanced devices and protocols have enabled high resolution acquisitions of a variety of anatomical structures with varying morphology across individuals or along time in longitudinal studies.

The specific problems that are encountered obviously depend on the nature of the applications and data. Yet, among the issues that are most often involved in such studies, one can mention in particular the quantification of shape differences through adequate metrics, registration (i.e. esti- mation transformations between two given shapes), or the estimation of shape statistics like means, templates or generative models from an observed population of shapes. $\mathrm{Nu}-$ merous methods have been proposed to address those questions for different shape modalities. Some of these methods, $[24,7,22,21]$ to mention only a few, rely on the extraction of a limited number of feature points (or landmarks). Other methods like [12, 32, 4, 3, 36] instead consider shapes as entire $2 \mathrm{D}$ or $3 \mathrm{D}$ images.

In many situations however, the more natural or compact model for shapes is to work with curves or surfaces of the ambient space which may have been obtained e.g. by prior segmentation of images. This case involves particular challenges resulting from the complex nature of spaces of curves and surfaces. The general goal of this paper is to propose a fairly general construction of similarity metrics between a wide class of those objects that can be easily used in multiple problems of shape analysis.

Relation to other works. Several frameworks have been introduced in the past to define similarity metrics or measures between two given curves or surfaces. If metrics like the Hausdorff or Gromov-Hausdorff distance [27] would appear natural at first glance, they are however highly sensitive to noise or topological irregularities and not necessarily fitted for applications to inexact registration.

A class of approaches have attempted to go through parametrization functions in order to represent and compare shapes with for example square root velocity functions for closed curves [35] or $q$-mappings for closed surfaces [25]. In both cases, enforcing parametrization-invariance is an essential step that involves delicate and possibly costly optimization over discrete reparametrizations.

The focus of this paper is rather on shape distances that are directly invariant to parametrization. The approach we 
follow stems from the idea from geometric measure theory of representing shapes as elements of a certain space of distributions, which was first used for the definition of shape similarity metrics in the seminal works of $[17,15]$. These were based on the representations of oriented curves or surfaces as mathematical currents. Later on, [11, 10] introduced the alternative but orientation-invariant representation known as varifolds before the higher order model of normal cycles was recently investigated in [31]. Since then, all these different frameworks have found numerous applications to the morphological analysis of cortical surfaces [26, 30], brain sulci [31], white matter fiber bundles [13, 18] or registration of lung vessels [29]...

Contributions. The objectives of this paper are several. We first propose to unify under one common and extended framework currents and varifolds' metrics for embedded curves and surfaces. This is based on an adaptation of the concept of oriented varifold; we show how this representation allows to construct a general class of shape similarity metrics that applies to any type of curve or surface (open or closed) or even reunions of multiple objects. Furthermore, these (Hilbert) metrics have the advantages of being independent to parametrization or to the sampling in the case of discrete shapes, efficiently computable with a closed-form expression and robust to small topological perturbations or potential segmentation issues. Besides the particular cases of currents and (unoriented) varifolds, we show that we can easily obtain from our construction new metrics with intermediate properties. We make a thorough comparison of the characteristics and behavior of a few distinct choices of those similarity measures and illustrate various possible uses. Our implementation is also freely available at [9].

\section{Notation and preliminaries}

In all the following, and although the framework we present can be extended to any dimension or codimension, we will restrict the exposition by considering shapes to be smooth manifolds or reunion of smooth manifolds (possibly with boundaries) of dimension 1 or 2 and embedded in the ambient space $\mathbb{R}^{n}$ with $n=2$ or $n=3$. These include most of the usual geometrical shapes in 2D or 3D like open and closed planar curves, 3D curves, surfaces, curve bundles...

Any such individual submanifold $X$ (curve or surface) inherits the Riemannian metric and volume measure denoted vol induced from the embedding Euclidean space. At each point $x \in X$, there exists a tangent space $T_{x} X$ which is a linear subspace of $\mathbb{R}^{n}$ of dimension 1 or 2 . An orientation of $X$ consists in giving an orientation to $T_{x} X$ for all $x \in X$ in which case each oriented tangent space can be represented as an element of an oriented Grassmannian. With shapes of dimension or codimension 1 , such oriented Grassmannian can be in both cases identified to the unit sphere $\mathbb{S}^{n-1}$ and $T_{x} X$ represented by the unit oriented tangent vector to the curve or unit oriented normal vector to the surface at $x$ that we shall write $\vec{t}(x)$. Note that the choice of the opposite orientation for the tangent space will change $\vec{t}(x)$ into $-\vec{t}(x)$. A shape is said to be orientable in the usual sense if there exists a smooth orientation application $x \mapsto \vec{t}(x)$ over the entire $X$.

\section{Shape representation as oriented varifolds}

\subsection{Representation of smooth submanifolds}

With the notation above, let $X$ be a smooth submanifold of $\mathbb{R}^{n}$ (curve or surface) with finite total volume $\operatorname{vol}(X)<$ $\infty$. In similar fashion as $[16,17,11]$, we associate to $X$ an oriented varifold $\mu_{X}$, i.e. a distribution on the space $\mathbb{R}^{n} \times \mathbb{S}^{n-1}$ of (position $\times$ tangent space orientation) and defined as follows:

$$
\mu_{X}(\omega)=\int_{X} \omega(x, \vec{t}(x)) d \operatorname{vol}(x)
$$

for any smooth test function $\omega: \mathbb{R}^{n} \times \mathbb{S}^{n-1} \rightarrow \mathbb{R}$. In the distribution sense, we may write $\mu_{X}=\int_{X} \delta_{(x, \vec{t}(x))} d \operatorname{vol}(x)$ with the Dirac delta $\delta_{(x, \vec{t}(x))}(\omega) \doteq \omega(x, \vec{t}(x))$ and the identification $X \rightarrow \mu_{X}$ gives indeed an injection from the set of smooth submanifolds to the dual $W^{*}$ of a certain space $W$ of test functions on $\mathbb{R}^{n} \times \mathbb{S}^{n-1}$ provided this latter space is large enough. Specific choices of spaces $W$ will be discussed with more details below.

Remark 1. The distribution $\mu_{X}$ only depends on the shape and not on any particular parametrization of $X$, but does depend a priori on the choice of orientation given to each tangent space through the vector $\vec{t}(x)$. In addition, the oriented varifold representation is additive in the sense that reunion of two distinct submanifolds $X$ and $Y$ satisfy $\mu_{X \cup Y}=\mu_{X}+\mu_{Y}$.

Remark 2. This general shape representation includes as particular cases both the previous frameworks of currents [16] and (unoriented) varifolds [11]. Each simply consists in restricting to spaces of test functions with particular forms as we will see hereafter.

\subsection{Polyhedral shapes' finite approximations}

Oriented varifolds can be then further used to embed within the same distribution space $W^{*}$ discrete curves or surfaces or reunions of those. Indeed, such discrete shapes are typically polyhedral objects which we can write in general as $X=\bigcup_{i=1}^{F} X_{i}$ where the cells $X_{i}$ are either line segments in the curve case or triangles in the case of $3 \mathrm{D}$ triangulated surfaces and are distinct to each other (modulo their boundaries).

Following Remark 1, we associate to such $X=\bigcup_{i=1}^{F} X_{i}$ the oriented varifold $\mu_{X}=\sum_{i=1}^{F} \mu_{X_{i}}$ where each $\mu_{X_{i}}$ is 

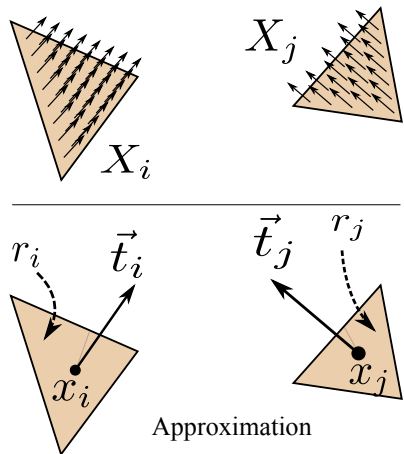

Figure 1. Oriented varifold approximation of triangulated faces.

the oriented varifold associated to the flat cell $X_{i}$ according to eq. (1). As illustrated by Figure 1, we can make the approximation:

$$
\mu_{X_{i}}(\omega) \approx \int_{X_{i}} \omega\left(x_{i}, \overrightarrow{t_{i}}\right) d \operatorname{vol}(x)=r_{i} \omega\left(x_{i}, \overrightarrow{t_{i}}\right)
$$

where $x_{i}$ is the barycenter of all vertices defining $X_{i}, \vec{t}_{i} \in$ $\mathbb{S}^{n-1}$ the orientation vector directing the linear subspace spanned by $X_{i}$ and $r_{i} \doteq \operatorname{vol}\left(X_{i}\right)$ the length (resp area) of the segment (resp triangle). This leads to replace each $\mu_{X_{i}}$ by a single weighted Dirac $r_{i} \cdot \delta_{\left(x_{i}, \vec{t}_{i}\right)}$. Then, writing $\tilde{\mu}_{X}=\sum_{i=1}^{F} r_{i} . \delta_{\left(x_{i}, \vec{t}_{i}\right)}$ one has $\mu_{X} \approx \tilde{\mu}_{X}$ with the following approximation bound:

Proposition 1. If $X$ is a shape contained inside the compact $K$ of $\mathbb{R}^{n}$ then for any $C^{1}$ test function $\omega$ :

$$
\left|\mu_{X}(\omega)-\tilde{\mu}_{X}(\omega)\right| \leq\left\|\partial_{x} \omega\right\|_{\infty, K} \cdot \operatorname{vol}(X) \cdot \max _{1 \leq i \leq F} \operatorname{diam}\left(X_{i}\right)
$$

Proof. For any cell $X_{i}$, since for all $x \in X_{i}, \vec{t}(x)=\vec{t}_{i}$, we have

$$
\begin{aligned}
\left|\left(\mu_{X_{i}}-r_{i} \delta_{\left(x_{i}, \vec{t}_{i}\right)}\right)(\omega)\right| & \leq \int_{X_{i}}\left|\omega(x, \vec{t}(x))-\omega\left(x_{i}, \vec{t}_{i}\right)\right| d \mathrm{vol} \\
& \leq \operatorname{vol}\left(X_{i}\right)\left\|\partial_{x} \omega\right\|_{K, \infty} \operatorname{diam}\left(X_{i}\right)
\end{aligned}
$$

with $\left\|\partial_{x} \omega\right\|_{\infty, K}$ denoting the sup-norm over $K$ of the derivatives of $\omega$ with respect to the first variable and $\operatorname{diam}\left(X_{i}\right)=\max _{x, y \in X_{i}}|x-y|$ the diameter of the cell. The result follows by just summing over all cells.

Therefore, the finite sum of Diracs $\tilde{\mu}_{X}$ provides an acceptable approximation of the polyhedral shape $X$ in terms of oriented varifolds as long as the size of cells remains small enough.

Another interesting property of this oriented varifold setting is that it provides consistency between discrete and continuous shape representations in the sense that the sequence of discrete distributions obtained from more and more refined polyhedral approximations of a given continuous curve or surface will converge, under certain technical assumptions, to the oriented varifold of the continuous shape. Precise conditions and proof could be generalized from similar recent results on currents and varifolds that have been derived in [28] and [1] but this would go beyond the scope of this paper. Yet an important consequence is that such representations are robust to discrete resampling.

\section{Kernel spaces and metric properties}

We will proceed in defining a class of metric structures on spaces of oriented varifolds that will in turn induce metrics between shapes. These dual metrics on $W^{*}$ are obtained by introducing more specific Hilbert spaces for the set of test functions $W$. For such spaces, Reproducing Kernel Hilbert Spaces (RKHS) turn out to be particularly well suited for practical computations on discrete shapes.

\subsection{RKHS of oriented varifolds}

We remind that a positive definite kernel on any set $M$ is a function $k: M \times M \rightarrow \mathbb{R}$ such that for any family $\left(x_{1}, \ldots, x_{p}\right) \in M^{p}$ of distinct points in $M$ the matrix $\left(k\left(x_{i}, x_{j}\right)\right)_{i, j=1, \ldots, p}$ is symmetric positive semidefinite. To any such kernel is associated, through Moore-Aronszajn theorem [2], a Hilbert space of functions on $M$ called the RKHS of $k$. Reproducing kernels have been vastly used for instance in machine learning [20].

In the present case, we wish to construct real kernels over the product space $\mathbb{R}^{n} \times \mathbb{S}^{n-1}$ and take the associated RKHS as the test functions' space $W$ in the oriented varifold representation. We consider, in this paper, a particular class of separable kernels defined as follows:

Proposition 2. If $k_{p o s}$ and $k_{\text {or }}$ are two positive definite kernels of class $C^{1}$ on $\mathbb{R}^{n}$ and $\mathbb{S}^{n-1}$ then the tensor product $k=k_{\text {pos }} \otimes k_{\text {or }}$ is a $C^{1}$ positive definite kernel on $\mathbb{R}^{n} \times \mathbb{S}^{n-1}$. In addition, the RKHS of $k$ is continuously embedded in $C^{1}\left(\mathbb{R}^{n} \times \mathbb{S}^{n-1}, \mathbb{R}\right)$.

This is a consequence of classical results on the tensor product of reproducing kernels (c.f [6] 1.4.6). The reproducing kernel property implies in addition that all Diracs $\delta_{(x, \vec{t})}$ belong to $W^{*}$ and that the dual metric satisfies

$$
\begin{aligned}
\left.\left\langle\delta_{\left(x_{1}, \vec{t}_{1}\right)}, \delta_{\left(x_{2}, \vec{t}_{2}\right)}\right)\right\rangle_{W^{*}} & =k\left(\left(x_{1}, \vec{t}_{1}\right),\left(x_{2}, \vec{t}_{2}\right)\right) \\
& =k_{\text {pos }}\left(x_{1}, x_{2}\right) k_{\text {or }}\left(\vec{t}_{1}, \vec{t}_{2}\right) .
\end{aligned}
$$

Although this separable structure does not cover the entire possible set of kernels on $\mathbb{R}^{n} \times \mathbb{S}^{n-1}$, the tensor product construction has the advantage of still providing a large class of metrics through the various possible choices of $k_{\text {pos }}$ and $k_{o r}$ while being easy to interpret in terms of the combination between spatial and orientation characteristics. Indeed, $k_{\text {pos }}$ can be thought as the measure of proximity between point positions in the embedding space $\mathbb{R}^{n}$ while $k_{o r}$, 
on the other hand, quantifies the proximity between the associated tangent spaces represented by vectors on the unit sphere.

An additional relevant property for the metric is the equivariance to rigid motion, namely that for any affine isometry $x \mapsto A x+b$ one has for any two Diracs in $W^{*}$ $\left\langle\delta_{\left(A x_{1}+b, A \vec{t}_{1}\right)}, \delta_{\left(A x_{2}+b, A \vec{t}_{2}\right)}\right\rangle_{W^{*}}=\left\langle\delta_{\left(x_{1}, \vec{t}_{1}\right)}, \delta_{\left(x_{2}, \vec{t}_{2}\right)}\right\rangle_{W^{*}}$. We have the following straightforward characterization:

Proposition 3. Within the previous class of separable kernels, the metric $W^{*}$ is equivariant to the action of rigid transformations for kernels $k$ of the form:

$$
\begin{aligned}
k\left(\left(x_{1}, \vec{t}_{1}\right),\left(x_{2}, \vec{t}_{2}\right)\right) & =k_{\text {pos }}\left(x_{1}, x_{2}\right) k_{o r}\left(\vec{t}_{1}, \vec{t}_{2}\right) \\
& =\rho\left(\left|x_{1}-x_{2}\right|\right) \gamma\left(\vec{t}_{1} \cdot \vec{t}_{2}\right) .
\end{aligned}
$$

Conversely, conditions on functions $\rho$ and $\gamma$ to obtain positive definite kernels have also been more precisely examined. The results of [33] have shown that admissible $\rho$ can be defined as the Bessel transform of a finite positive Borel measure on $\mathbb{R}_{+}$. Similarly, a necessary and sufficient condition on $\gamma$ (cf [37]) is that $\gamma(u)=\sum_{k=0}^{\infty} a_{k} P_{k}^{(\lambda)}(u)$ with $a_{k} \geq 0, \sum_{k=0}^{\infty} a_{k} P_{k}^{(\lambda)}(1)<\infty$ and $P_{k}^{(\lambda)}$ the ultraspherical polynomials of order $\lambda=(n-1) / 2$. Note that another possible (but not exhaustive) way of constructing admissible $\gamma$ is by restriction to the unit sphere of radial kernels on $\mathbb{R}^{n}$.

A particular subclass of the kernels of Proposition 3 are the ones such that $\gamma(u)=u$ and that correspond to the framework of currents. Another subclass are the ones invariant to reorientation i.e. such that $\gamma(-u)=\gamma(u)$ (or $a_{k}=0$ for odd $k$ in the decomposition with ultraspherical polynomials) which results in metrics on $W^{*}$ also independent on the orientation: this precisely corresponds to the framework of unoriented varifolds developed previously in $[11,14]$, cf Section 4.3 for examples.

\subsection{Metrics induced on shapes}

We can now restrict the metric on $W^{*}$ defined in 4.1 to shapes through the identification of $X$ as $\mu_{X} \in W^{*}$. However, this identification may not be injective if the space $W$ is too 'small' which may only result in a pseudo-metric between shapes. A sufficient condition is the following:

Proposition 4. Let $k_{\text {pos }}$ and $k_{\text {or }}$ be two kernels as in Proposition 2 such that in addition $k_{\text {pos }}$ is a $C_{0}$-universal kernel on $\mathbb{R}^{n}$ and for all $\vec{t} \in \mathbb{S}^{n-1}, k_{\text {or }}(\vec{t}, \vec{t})>0$. Then $d_{W^{*}}(X, Y)=\left\|\mu_{X}-\mu_{Y}\right\|_{W^{*}}$ defines a distance on the set of shapes.

Proof. We only need to show that $d_{W^{*}}(X, Y)=0 \Rightarrow$ $X=Y$. Let's write $W_{\text {pos }}$ and $W_{\text {or }}$ for the RKHS on $\mathbb{R}^{n}$ and $\mathbb{S}^{n-1}$ associated to kernels $k_{\text {pos }}$ and $k_{\text {or }}$. The $C_{0^{-}}$ universality property of $k_{\text {pos }}$ precisely means that $W_{\text {pos }}$ is dense in the space of continuous functions on $\mathbb{R}^{n}$ vanishing at $\infty$ [8]. Now, let's assume that $X$ and $Y$ are both smooth curves or surfaces (the case of reunions of those can be treated similarly) such that $d_{W^{*}}(X, Y)=0$ and $X \neq Y$. Then there exist $x_{0} \in X$ and $r>0$ such that $B\left(x_{0}, r\right) \cap Y=\emptyset$. Consider the function $g$ defined by $g(\cdot)=k_{\text {or }}\left(\vec{t}\left(x_{0}\right), \cdot\right) \in W_{\text {or }}$. Since $g\left(\vec{t}\left(x_{0}\right)\right)=$ $k_{\text {or }}\left(\vec{t}\left(x_{0}\right), \vec{t}\left(x_{0}\right)\right)>0, g$ is continuous and $X$ is smooth, $x \mapsto g(\vec{t}(x))$ is strictly positive on a certain ball $B\left(x_{0}, r^{\prime}\right) \cap$ $X \subset B\left(x_{0}, r\right)$. Now take $h$ a continuous positive function on $\mathbb{R}^{n}$ supported in $B\left(x_{0}, r^{\prime}\right)$ and strictly positive at $x_{0}$. By density, there exists a sequence $\left(h_{n}\right) \in\left(W_{\text {pos }}\right)^{\mathbb{N}}$ converging uniformly to $h$. Then

$$
\begin{aligned}
0 & =\left(\mu_{X}-\mu_{Y}\right)\left(h_{n} \otimes g\right) \\
& =\int_{X} h_{n}(x) g(\vec{t}(x)) d \operatorname{vol}(x)-\int_{Y} h_{n}(y) g(\vec{t}(y)) d \operatorname{vol}(y) \\
& \stackrel{n \rightarrow \infty}{\longrightarrow} \int_{X \cap B\left(x_{0}, r^{\prime}\right)} h(x) g(\vec{t}(x)) d \operatorname{vol}(x) .
\end{aligned}
$$

But since $h$ and $x \mapsto g(\vec{t}(x))$ are both strictly positive on $B\left(x_{0}, r^{\prime}\right)$, we end up with a contradiction.

Thanks to both eq. (1) and the reproducing kernel property of eq. (3), for any two submanifolds $X$ and $Y$, we have the following simple and explicit expression for the metric:

$$
\begin{aligned}
& \left\langle\mu_{X}, \mu_{Y}\right\rangle_{W^{*}} \\
& =\iint_{X \times Y} k_{p o s}(x, y) k_{o r}(\vec{t}(x), \vec{t}(y)) d \operatorname{vol}^{X}(x) d \operatorname{vol}^{Y}(y) .
\end{aligned}
$$

Note that the induced distance $d_{W^{*}}$ inherits the equivariance to rigid motion for kernels chosen as in Proposition 3, i.e. that for any affine isometry $S$, one has $d_{W^{*}}(S(X), S(Y))=d_{W^{*}}(X, Y)$.

Similarly, with the discrete approximations of polyhedral shapes described in Section 3.2, we obtain a finite equivalent of (5) that writes:

$$
\left\langle\tilde{\mu}_{X}, \tilde{\mu}_{Y}\right\rangle_{W^{*}}=\sum_{i=1}^{F^{X}} \sum_{j=1}^{F^{Y}} k_{p o s}\left(x_{i}, y_{j}\right) k_{o r}\left(\vec{t}_{i}^{X}, \vec{t}_{j}^{Y}\right) r_{i}^{X} r_{j}^{Y}
$$

for the two discretizations $\tilde{\mu}_{X}=\sum_{i=1}^{F^{X}} r_{i}^{X} \delta_{\left(x_{i}, \vec{t}_{i}^{X}\right)}$ and $\tilde{\mu}_{Y}=\sum_{j=1}^{F^{Y}} r_{j}^{Y} \delta_{\left(y_{j}, \vec{t}_{j}^{Y}\right)}$. Note that thanks to the $C^{1}$ regularity of both kernels, it is straightforward to deduce from Proposition 1 a similar bound on the approximation error for the $W^{*}$ metric, i.e. that $\left\|\mu_{X}-\tilde{\mu}_{X}\right\|_{W^{*}}$ is again controlled by the max diameter of all cells in $X$. A second consequence of the regularity is that the metric of (6) is differentiable with respect to the positions of shapes' vertices and the gradient is easily computed by chain rule differentiation. 


\subsection{Choosing kernels}

From now on, we focus the discussion on kernels of the form given by (4). The particularly simple form of this family of metrics makes it very convenient for an implementation that preserves the flexibility in the choices of kernels.

For the radial kernel $k_{\text {pos }}$, typical choices are the widely used Gaussian $k_{p o s}(x, y)=e^{-\frac{|x-y|^{2}}{\sigma^{2}}}$ or Cauchy $k_{\text {pos }}(x, y)=\frac{1}{1+|x-y|^{2} / \sigma^{2}}$ kernels in which parameter $\sigma$ gives the spatial scale at which the metric is sensitive. Both satisfy the $C_{0}$-universality assumption of Proposition 4 , cf [8]. We shall be mostly experimenting with Gaussian kernels in the simulations of next section. Our implementation also includes sums of arbitrary number of Gaussians with different scales as an attempt to allow multiscale strategies.

The equivariant spherical kernel $k_{o r}$ on the other hand has deep influence on the fundamental properties of the resulting metrics. Below, we examine several distinctive examples for $\gamma$, some of which retrieve, as special cases, previously introduced shape dissimilarity terms:

- $\gamma(u)=1$ (distributions): gives metrics that discard the orientation vector information to only evaluate the relative positions between points in the two shapes. Those metrics can be equivalently obtained from the representation of shapes as standard distributions on $\mathbb{R}^{n}$, which was the initial approach of [16].

- $\gamma(u)=u$ (currents): $k_{o r}$ is the restriction of the linear kernel on $\mathbb{S}^{n-1}$. Because of linearity, $\mu_{X}$ can be interpreted as an element of the dual of the space of vector fields or in other words as a current and the family of metrics (5) recovers exactly the framework introduced in $[17,15]$. Such metrics are only well-suited for orientable shapes. Given a surface or curve $X$, we denote $\check{X}$ the same surface or curve but oppositely oriented. The linearity of the kernel implies in that case that $\mu_{\check{X}}=-\mu_{X}$.

- $\gamma(u)=u^{2}$ (unoriented varifolds): corresponds to the Binet kernel on $\mathbb{S}^{n-1}$, invariant to orientation. This is the simplest example of unoriented varifold metrics used in [14]. Other orientation-invariant kernels have also been proposed and studied in [11].

- $\gamma(u)=e^{\frac{2 u}{\sigma_{s}^{2}}}$ (oriented varifolds): in that case, $k_{o r}$ is the extrinsic Gaussian kernel on the sphere (the restriction of the Gaussian kernel of $\mathbb{R}^{n}$ to $\mathbb{S}^{n-1}$ ). The shapes need to be orientable and the kernel is sensitive to the orientation but non-linear, the scale $\sigma_{s}$ enforces the angular sensitivity of the metric. Many other kernels with similar properties can be used, in particular the intrinsic heat kernels on $\mathbb{S}^{n-1}$ although its expression involves decomposition into the ultraspherical polynomial basis which is thus more numerically intensive.
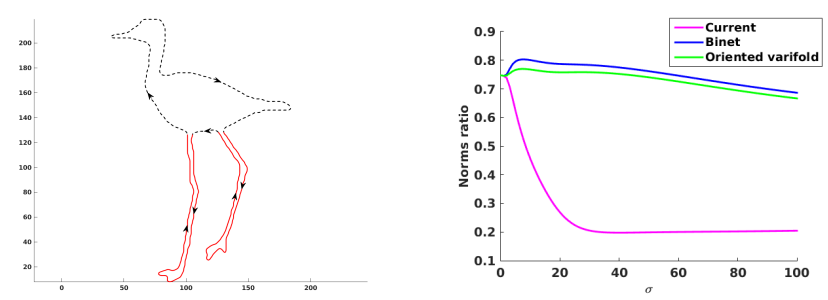

Figure 2. Behavior of the different metrics. On left, a shape with thin elongated components in red. On right, a plot of the ratios between the norm of the red part with the norm of the entire shape for increasing scales $\sigma$ for the Gaussian function $\rho$. Note the rapid decrease in the case of currents, due to the cancellation of oppositely oriented pieces 'close' to each other with respect to $\sigma$.

With both varifold representations, the non-linearity of $k_{\text {or }}$ prevents cancellations of thin structures when compared to currents, see Figure 2. Conversely, this linearity property enforces more regularity in some specific registration problems to model shape growth [23].

\section{Experiments}

We now give several illustrations of the respective interests and drawbacks of these different metrics, in particular the novel oriented varifolds' metric, on a few problems related to shape analysis and processing. We use the specific kernels described in Section 4.3 for currents, unoriented and oriented varifolds.

\subsection{Shape clustering}

In this section, we present the experimental results for object retrieval on the standard shape dataset Kimia-216. It consists in 18 classes with 12 objects in each. The contour of each shape is extracted from its smoothed image. We do not control the final sample of each contour. Curves have between 100 and 305 points. To address the question of scale invariance, we fix an oriented varifold's metric in accordance with the average scale of the data set and we apply a scaling to all the shapes such that, in fine, they all have the same norm. From the previous equivariant metrics on $W^{*}$, we then obtain a rigid-invariant metrics on shapes by solving the rigid registration problem:

$$
d_{\text {inv }}(X, Y)=\inf _{S \text { rigid }} d_{W^{*}}(X, S(Y)) .
$$

The top 11 closest matches, obtained with the rigidinvariant current, oriented and unoriented varifolds' metrics, are shown in Table 5.1. The metrics are defined with a Gaussian radial kernel for $k_{\text {pos }}$, which depends on the scale parameter $\sigma$ and the three scalar functions $\gamma$ introduced in Section 4.2, the last of which depends on an additional parameter $\sigma_{s}$. We tested the classification for a small set of different parameters. The optimal results were ob- 


\begin{tabular}{|l|c|c|c|c|c|c|c|c|c|c|c|c|}
\hline Method & $1^{\text {st }}$ & $2^{\text {nd }}$ & $3^{\text {rd }}$ & $4^{\text {th }}$ & $5^{\text {th }}$ & $6^{\text {th }}$ & $7^{\text {th }}$ & $8^{\text {th }}$ & $9^{\text {th }}$ & $10^{\text {th }}$ & $11^{\text {th }}$ & Total \\
\hline \hline Shape context [5] & 214 & 209 & 205 & 197 & 191 & 178 & 161 & 144 & 131 & 101 & 78 & 76.13 \\
Shock graph [34] & 216 & 216 & 216 & 215 & 210 & 210 & 207 & 204 & 200 & 187 & 163 & 94.44 \\
Chordal axis transform [38] & 216 & 216 & 216 & 214 & 215 & 212 & 213 & 208 & 204 & 195 & 168 & 95.83 \\
Current & 214 & 211 & 205 & 202 & 200 & 203 & 197 & 197 & 189 & 160 & 150 & 89.56 \\
Unoriented varifold & 214 & 216 & 212 & 210 & 210 & 207 & 202 & 201 & 191 & 171 & 150 & 91.92 \\
Oriented varifold & 215 & 214 & 214 & 213 & 212 & 206 & 206 & 202 & 192 & 185 & 161 & 93.43 \\
\hline
\end{tabular}

Table 1. Top 11 closest matching shapes for Kimia-216. The oriented varifold representation outperforms the current and unoriented varifold representations, with a total retrieval result of $93.43 \%$ in the nearest 11 matches.

\begin{tabular}{|l|c|}
\hline Method & Retrieval accuracy \\
\hline \hline Current & 96.30 \\
Unoriented varifold & 96.57 \\
Oriented varifold & 97.92 \\
\hline
\end{tabular}

Table 2. Bull Eye Score (retrieval rate among the 24 nearest neighbors, query included) of proposed methods for Kimia-216.

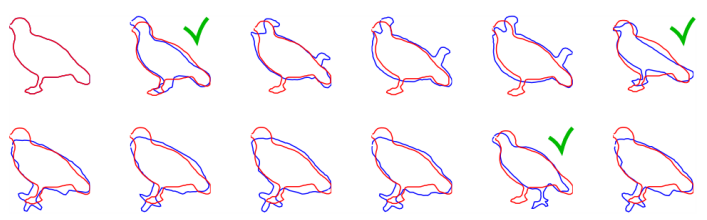

Figure 3. Least accurate top 12 retrieval of a query shape.

tained with $\sigma=1.5$ and $\sigma_{s}=.5$ for oriented varifolds, $\sigma=1.5$ for unoriented varifolds and $\sigma=1$ for currents.

The final score of the classification is significantly reduced by few queries with a particularly low retrieval score as displayed in Figure 3.

\subsection{Diffeomorphic registration}

\subsubsection{The deformation model}

As a second class of applications, we turn to the use of the previous metrics as data fidelity terms for diffeomorphic registration algorithms of curves and surfaces. The framework we propose is sufficiently flexible to be embedded in a variety of inexact registration methods; in this paper, we focus on the Large Deformation Diffeomorphic Metric Mapping (LDDMM) model described in [4, 39].

In this model, diffeomorphisms are constructed as flows of time-dependent velocity fields $t \in[0,1] \mapsto v_{t}$, each $v_{t}$ belonging to a predefined Hilbert space $V$ of smooth vector fields. If $X_{0}$ denotes the template shape and $X_{1}$ the target, then the registration of $X_{0}$ to $X_{1}$ is written as the minimization problem:

$$
\min _{v \in L^{2}([0,1], V)}\left\{\int_{0}^{1}\left\|v_{t}\right\|_{V}^{2} d t+\lambda\left\|\mu_{\phi_{1}^{v}\left(X_{0}\right)}-\mu_{X_{1}}\right\|_{W^{*}}^{2}\right\}
$$

where $\phi_{1}^{v}\left(X_{0}\right)$ is the deformed template by $\phi_{1}^{v}$ obtained by

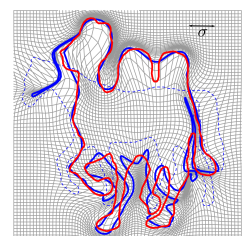

Current

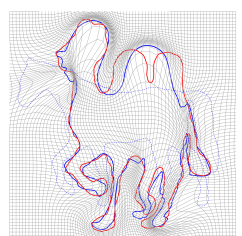

Unor varifold

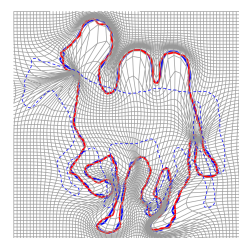

Or varifold
Figure 4. Diffeomorphic registration using the different metrics of a template horse (blue dotted curve) to the target (in red). The estimated deformed template is shown in blue together with deformation grid. Kernel $k_{\text {pos }}$ is in each case a Gaussian with spatial scale $\sigma=15$.

the flow equation $\partial_{t} \phi_{t}^{v}=v_{t} \circ \phi_{t}^{v}$ with $\phi_{0}^{v}=\operatorname{Id}$ and $\lambda>0$ is a weight parameter between the regularization and data fidelity terms. This problem is numerically solved for discrete shapes through a geodesic shooting procedure that involves the computation of both oriented varifold distances and their gradient with respect to vertex positions.

\subsubsection{Registration of closed curves}

We first compare registration results on (oriented) closed curves for current, unoriented and oriented varifolds. Figure 4 shows the template, target and registered shape as well as the final deformation grid in each case. With currents, notice the apparition of degenerate structures as well as the fact that the two humps are not well recovered: this is another manifestation of the cancellation effect previously mentioned. These effects are totally avoided in the two other cases. However, unoriented varifolds, by being insensitive to the orientation, tend to have more difficulties in reproducing the convoluted double hump by instead creating a single 'average' hump. Oriented varifolds achieve very accurate registration in that case.

\subsubsection{Registration of set of curves}

We consider now a simple example involving disconnected 'bags' of curves instead of the previous single connected shapes. These were obtained by extracting sketches from two images of faces using the Line Segment Detector al- 

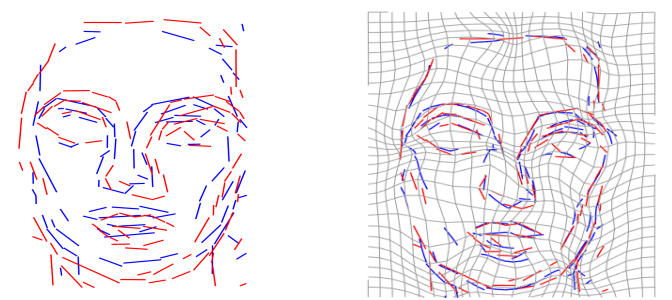

Figure 5. Registration between two face sketches using unoriented varifolds.

gorithm of [19]. In this situation, orienting consistently each separate segment is obviously a very cumbersome issue. Yet the unoriented varifold metric allows to compare those objects without requiring that orientation step.

\subsubsection{Registration of noisy shapes}

Data are often observed with an additive noise. In that situation, the cancellation property of currents can be used advantageously to suppress the effects of noise in the estimated deformation as shown in Figure 6 and 7. This holds for kernel sizes $\sigma$ chosen sufficiently large compared to noise induced oscillations. On the other hand, the oriented and unoriented varifold approaches are consistently much more affected by noise. Note, however, that if both the template and the target present similar type and level of noise, all three approaches will generally lead to good registrations.

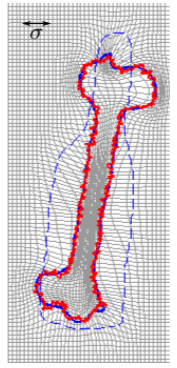

Current

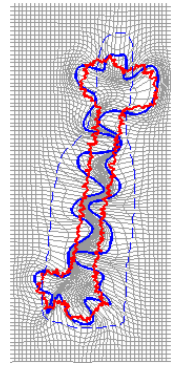

Unoriented varifold

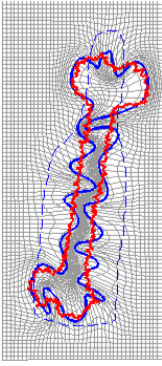

Oriented varifold

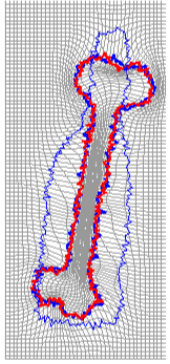

Noisy template
Figure 6. Registration with noisy target. Note that non-linear kernels (oriented or not) are much more sensitive to noise as opposed to currents. In the last experiment, noise is also added to the template and the results are similar for the three metrics.

\subsubsection{Registration of multi-objects}

Multi-modal data analysis is a common problem in medical imaging. We present therefore in Figure 8 an example of registration involving multi-shapes that mix objects of various types. The source and target shapes contain each a surface (a hand with 5000 and 5055 triangles respectively)
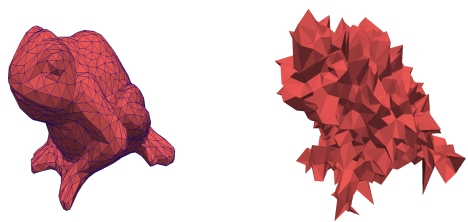

Smooth surface

Noisy version
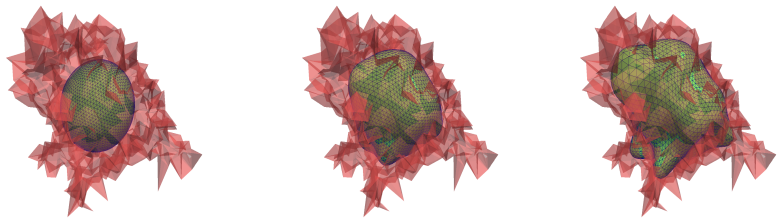

Deformation: $t=0$

$$
t=0.3
$$$$
t=0.6
$$

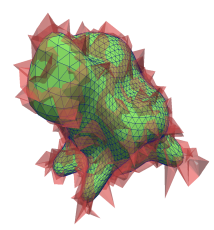

$\mathrm{t}=1$

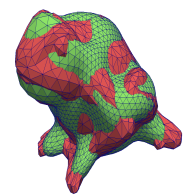

Overlap with noise-free surface

Figure 7. Registration of a sphere (2560 vertices) on a noisy surface (1165 vertices) using currents. The template shape evolution along the deformation is shown as well as the comparison of the registered shape and the initial noise-free surface.

and a bundle of curves (50 and 53 curves respectively containing 9 points each). To fix the notation, $X=\left(X^{1}, X^{2}\right)$ denotes the source multi-shape and $Y=\left(Y^{1}, Y^{2}\right)$ denotes the target multi-shape where the first object corresponds to the surface part and the second one to the curve part.

To register two of these multi-shapes, we consider a variant of the optimization problem of (8) that writes

$$
\begin{aligned}
\min _{v \in L^{2}([0,1], V)}\{ & \int_{0}^{1}\left\|v_{t}\right\|_{V}^{2} d t+\lambda_{1}\left\|\mu_{\phi_{1}^{v}\left(X_{1}\right)}-\mu_{Y_{1}}\right\|_{W_{1}^{*}}^{2} \\
& \left.+\lambda_{2}\left\|\mu_{\phi_{1}^{v}\left(X_{2}\right)}-\mu_{Y_{2}}\right\|_{W_{2}^{*}}^{2}\right\},
\end{aligned}
$$

where $\lambda_{1}, \lambda_{2}>0$ are weighting parameters. It is important to properly calibrate the $\lambda_{i}$ 's as the order of magnitude of the two varifold data attachment terms may be very different. Finally note that the deformation $\phi_{1}^{v}$ is common to the two sub-shapes, whereas the data attachment term is specific to each sub-shape.

\subsubsection{Registration onto an incomplete shapes}

Kernel-based oriented varifold distances coupled with the LDDMM framework introduced Section 5.2.1 can be readily used even when the mesh of the data at hand has missing parts.

In our experiments, we assume that the source shape is complete whereas the target shape has a proportion $(1-p)$ 

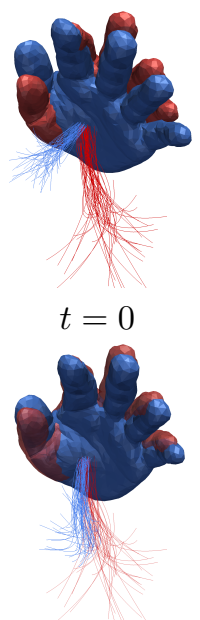

$t=3 / 5$
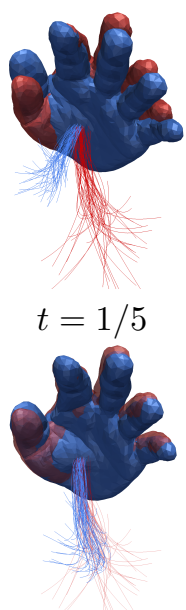

$t=4 / 6$
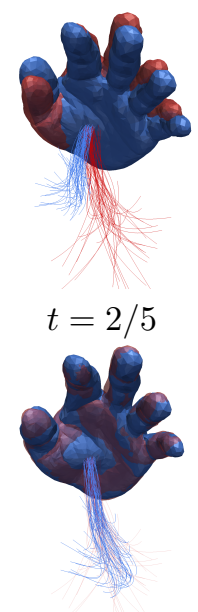

$t=1$
Figure 8. Registration, with unoriented varifolds, of multi-shapes containing each a surface and a set of curves. Target shape is in red.

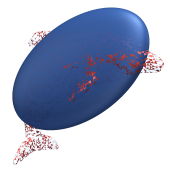

$t=0$

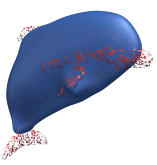

$$
t=1 / 3
$$

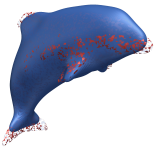

$t=2 / 3$

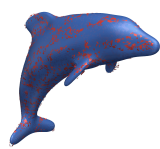

$t=1$
Figure 9. Registration of an ellipsoid on a dolphin with $70 \%$ missing triangles, using oriented varifold data attachment term.

of missing triangles. For instance, in Figure 9 the target dolphin mesh contains $p=30 \%$ of triangles of the complete mesh (originally containing 14207 triangles). The removed faces were picked uniformly at random. The source shape is an ellipsoid containing 20000 triangles. To give some perspective on the numerical cost, computing the oriented varifold distance between the source and the complete target typically takes 0.1 second with our CUDA implementation on a Nvidia Geforce GTX 780 Ti.

In order to get a coherent registration on such an incomplete target, it is, however, necessary to adjust for the deficiency in total area of the target. The orientation varifold representation allows to do so very conveniently by simply re weighting the target oriented varifold with a single constant equal to an estimation of the proportion of missing triangles. Then the registration problem is replaced by:

$$
\min _{v \in L^{2}([0,1], V)}\left\{\int_{0}^{1}\left\|v_{t}\right\|_{V}^{2} d t+\lambda\left\|\mu_{\phi_{1}^{v}(X)}-\frac{\mu_{Y}}{p}\right\|_{W^{*}}^{2}\right\}
$$

where $(1-p)$ is the proportion of missing triangles in the target shape $Y$. Figure 10 shows some experiments where the proportion $p$ is known. Registration results look globally good even for small $p$ and seem almost perfect for any $p$ greater than $30 \%$. Below this value, the registration start
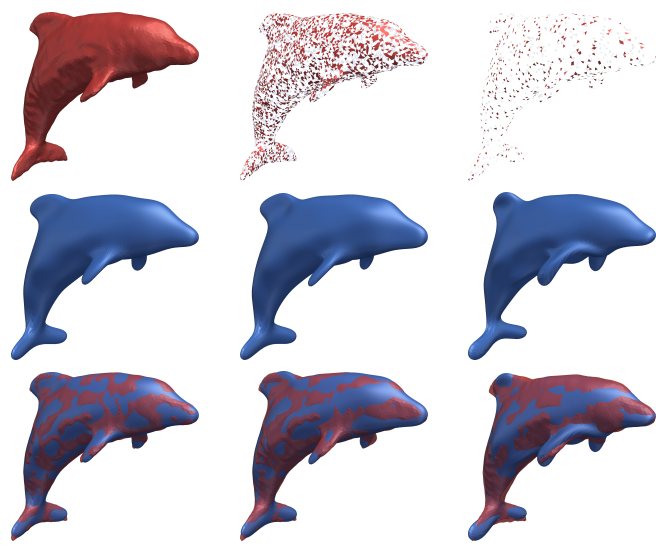

$p=100 \%$

$$
p=5 \%
$$

Figure 10. Three registrations onto a target with various ratios of missing faces. The proportion $(1-p)$ of missing triangles is known. Corresponding target shapes are on the first row. Deformed ellipsoids are plotted on the second row. Comparison between the deformed ellipsoid and the complete target are shown on the third row.

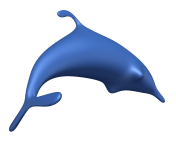

$$
p=1
$$

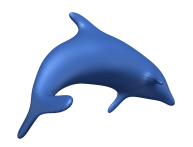

$p=0.7$

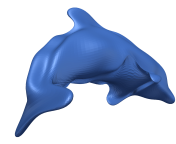

$p=0.5$

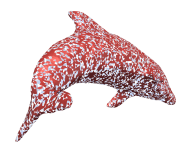

target
Figure 11. Registered ellipsoid onto a dolphin with missing triangles as in Figure 9. The true proportion of triangles of the target is $p^{*}=0.6$. When $p>p^{*}$ (resp. $p<p^{*}$ ) the resulting matching looks thinner (resp. bulgy) due to the difference in total area.

to slightly deteriorate and spurious deformations may affect the mesh in area with complex geometries. If the true proportion $p^{*}$ is unknown, the quality of the registration is affected by the value of the chosen $p$ as shown in Figure 11.

\section{Conclusion}

In this paper, we have proposed a general framework for 2D and 3D shape similarity measures, invariant to parametrization and equivariant to rigid transformations. In this simple and efficient framework that encompasses currents and unoriented varifolds, the choice of the metric reduces to scalar functions with only one or two scale parameters that parametrize families of kernels. A numerical toolbox is freely available at [9]. It includes all the functions introduced in this paper and allows to readily define new customized kernel metrics.

We illustrated through numerous examples that these shape representations and associated metrics are well-suited for large classes of data structures and highlighted the specific features for different sub-categories of those metrics. Beyond the use as fidelity terms for registration, we wanted to emphasize how such a framework could be also directly applied in shape clustering or classification problems. 


\section{References}

[1] S. Arguillère, E. Trélat, A. Trouvé, and L. Younes. Registration of Multiple Shapes using Constrained Optimal Control. SIAM Journal on Imaging Sciences, 9(1):344-385, 2016. 3

[2] N. Aronszajn. Theory of reproducing kernels. Trans. Amer. Math. Soc., 68:337-404, 1950. 3

[3] J. Ashburner. A fast diffeomorphic image registration algorithm. Neuroimage, 38(95-113), 2007. 1

[4] M. F. Beg, M. I. Miller, A. Trouvé, and L. Younes. Computing large deformation metric mappings via geodesic flows of diffeomorphisms. International journal of computer vision, 61(139-157), 2005. 1, 6

[5] S. Belongie, J. Malik, and J. Puzicha. Shape matching and object recognition using shape contexts. IEEE Transactions on Pattern Analysis and Machine Intelligence, 24(4):509522, Apr 2002. 6

[6] A. Berlinet and C. Thomas-Agnan. Reproducing Kernel Hilbert Spaces in Probability and Statistics. Springer, 2004. 3

[7] F. L. Bookstein. Principal warps: thin-plate splines and the decomposition of deformations. IEEE Transactions on Pattern Analysis and Machine Intelligence, 11(6):567-585, 1989. 1

[8] C. Carmeli, E. De Vito, A. Toigo, and V. Umanita. Vector valued reproducing kernel Hilbert spaces and universality. Analysis and Applications, 8(01):19-61, 2010. 4, 5

[9] B. Charlier, N. Charon, and A. Trouvé. A short introduction to the functional shapes toolkit. https: / / github. com/ fshapes/fshapesTk/, 2014-2015. 2, 8

[10] N. Charon. Analysis of geometric and functional shapes with extensions of currents. Application to registration and atlas estimation. PhD thesis, ENS Cachan, 2013. 2

[11] N. Charon and A. Trouvé. The varifold representation of non-oriented shapes for diffeomorphic registration. SIAM journal of Imaging Sciences, 6(4):2547-2580, 2013. 2, 4, 5

[12] P. Dupuis, U. Grenander, and M. I. Miller. Variational problems on flows of diffeomorphisms for image matching. Quarterly of applied mathematics, 56(3):587, 1998. 1

[13] S. Durrleman, P. Fillard, X. Pennec, A. Trouvé, and N. Ayache. Registration, atlas estimation and variability analysis of white matter fiber bundles modeled as currents. NeuroImage, 55(3):1073-1090, 2010. 2

[14] S. Durrleman, M. Prastawa, N. Charon, J. Korenberg, S. Joshi, G. Gerig, and A. Trouvé. Deformetrics : morphometry of shape complexes with space deformations. Neuroimage, 101:35-49, 2014. 4, 5

[15] J. Glaunès, A. Qiu, M. Miller, and L. Younes. Large deformation diffeomorphic metric curve mapping. Int J Comput Vis, 80(3):317336, 2008. 2, 5

[16] J. Glaunès, A. Trouvé, and L. Younes. Diffeomorphic matching of distributions: A new approach for unlabelled pointsets and sub-manifolds matching. CVPR, 2:712-718, 2004. 2,5

[17] J. Glaunès and M. Vaillant. Surface matching via currents. Proceedings of Information Processing in Medical Imaging (IPMI), Lecture Notes in Computer Science, 3565(381-392), 2006. 2,5
[18] P. Gori, O. Colliot, L. Marrakchi-Kacem, Y. Worbe, F. D. V. Fallani, M. Chavez, C. Poupon, A. Hartmann, N. Ayache, and S. Durrleman. Parsimonious Approximation of Streamline Trajectories in White Matter Fiber Bundles. IEEE Transactions on Medical Imaging, PP(99), 2016. 2

[19] R. Grompone von Gioi, J. Jakubowicz, J.-M. Morel, and G. Randall. LSD: a Line Segment Detector. Image Processing On Line, 2:35-55, 2012. 7

[20] T. Hastie, R. Tibshirani, and J. Friedman. The elements of statistical learning. Springer, 2001. 3

[21] D. Holm, T. Ratnanather, A. Trouvé, and L. Younes. Soliton Dynamics in Computational Anatomy. Neuroimage, 23(S1):170-178, 2007. 1

[22] S. Joshi and M. I. Miller. Landmark matching via large deformation diffeomorphisms. Image Processing, IEEE Transactions on, 9(8):1357-1370, 2000. 1

[23] I. Kaltenmark. Geometrical Growth Models for Computational Anatomy. PhD thesis, ENS Paris-Saclay, 2016. 5

[24] D. Kendall. Shape manifolds, procrustean metrics, and complex projective spaces. Bulletin of the London Mathematical Society, 16(2):81-121, 1984. 1

[25] S. Kurtek, E. Klassen, Z. Ding, S. W. Jacobson, J. L. Jacobson, M. J. Avison, and A. Srivastava. Parameterizationinvariant shape comparisons of anatomical surfaces. IEEE Transactions on Medical Imaging, 30(3):849-858, 2011. 1

[26] J. Ma, M. I. Miller, and L. Younes. A Bayesian generative model for surface template estimation. Journal of Biomedical Imaging, 2010:16, 2010. 2

[27] F. Memoli. On the use of Gromov-Hausdorff Distances for Shape Comparison. Symposium on Point Based Graphics, pages 81-90, 2007. 1

[28] G. Nardi, B. Charlier, and A. Trouvé. The matching problem between functional shapes via a BV-penalty term: a $\Gamma$ convergence result. CoRR, abs/1503.07685, March 2015. 3

[29] Y. Pan, G. Christensen, O. Durumeric, S. Gerard, J. Reinhardt, and G. Hugo. Current- and Varifold-Based Registration of Lung Vessel and Airway Trees. CVPR, 2016. 2

[30] I. Rekik, G. Li, W. Lin, and D. Shen. Multidirectional and Topography-based Dynamic-scale Varifold Representations with Application to Matching Developing Cortical Surfaces. Neurolmage, 135:152-162, 2016. 2

[31] P. Roussillon and J. Glauns. Kernel Metrics on Normal Cycles and Application to Curve Matching. SIAM Journal on Imaging Sciences, 9(4):1991-2038, 2016. 2

[32] D. Rueckert, L. I. Sonoda, C. Hayes, D. L. G. Hill, M. O. Leach, and D. J. Hawkes. Nonrigid registration using freeform deformations: application to breast MR images. IEEE Transactions on Medical Imaging, 18(8):712-721, 1999. 1

[33] I. J. Schoenberg. Metric spaces and completely monotone functions. Annals of Mathematics, 39(4):811-841, 1938. 4

[34] T. B. Sebastian, P. N. Klein, and B. B. Kimia. Recognition of shapes by editing their shock graphs. IEEE Transactions on Pattern Analysis and Machine Intelligence, 26(5):550-571, May 2004. 6

[35] A. Srivastava, E. Klassen, S. H. Joshi, and I. H. Jermyn. Shape analysis of elastic curves in euclidean spaces. IEEE Transactions on Pattern Analysis and Machine Intelligence, 33(7):1415-1428, 2011. 1 
[36] T. Vercauteren, X. Pennec, A. Perchant, and N. Ayache. Diffeomorphic demons: Efficient non-parametric image registration . Neurolmage, 45(1):61-72, 2009. Mathematics in Brain Imaging. 1

[37] Y. Xu and E. W. Cheney. Strictly positive definite functions on spheres. Proceedings of the American Mathematical Society, 116(4):977-981, 1992. 4

[38] Z. Yasseen, A. Verroust-Blondet, and A. Nasri. Shape matching by part alignment using extended chordal axis transform. Pattern Recognition, 57:115 - 135, 2016. 6

[39] L. Younes. Shapes and diffeomorphisms. Springer, 2010. 6 